vascular smooth muscle cells. GSPE significantly reduced the AGEs $(p<0.05)$, and the expression of RAGE in aorta of diabetic rats. The expression of 23 proteins was found either up-regulated or downregulated in the aorta of untreated diabetic rats. Only the expression of 15 proteins was found either down-regulated or up-regulated in the aorta of GSPE treated diabetic rats. Among these proteins, in comparison with the aortic tissue of diabetic rats, the differential proteomic analysis of the aortic tissue of diabetic rats, treated by GSPE further revealed the variation of fifteen proteins, namely, lamin A, ATP synthase alpha chain, proline arginine-rich end leucine-rich repeat protein precursor, LOC500183 protein, heat Shock Protein 27, enoylCoA hydratase, glutamate dehydrogenase, protein-L-isoaspartate (Daspartate) O-methyltransferase 1, lactadherin, leucine aminopeptidase 3, adenylyl cyclase- associated protein 1, apolipoprotein A-I, catalase, Dermcidin, and fibrinogen $\beta$ chain. In brief, the differentially expressed proteins were related to many important biological functions including metabolism, oxidative stress, signal transduction, cell proliferation, cell growth, apoptosis and heat shock.

Conclusion GSPE plays an important role against diabetic macrovascular complications. Our findings might help to better understanding of the mechanism of diabetic macrovascular complications, and provide novel targets for estimating the effects of GSPE therapy.

\section{e0022 LOSARTAN ATTENUATED CARDIOMYOCYTE APOPTOSIS BY INCREASING AKT ACTIVITY IN AORTIC BANDED RATS WITH CHRONIC HEART FAILURE}

doi:10.1136/hrt.2010.208967.22

Deming Fu, Chuanshi Xiao, Yingru Chai. The Second Hospital of Shanxi Medical University

Objective The present study was undertaken to investigate the protective effects of losartan on cardiomyocyte apoptosis in chronic heart failure (CHF) rats induced by banding abdominal aorta.

Methods SD rats underwent abdominal aorta coarctation to induce CHF, confirmed by ultrasound cardiograph and Catheterisation, or sham operation, followed by 8 weeks treatment with Losartan or vehicle. Plasma NE was measured by ELISA, and plasma and tissue Ang II levels were measured by RIA. Cardiomyocyte apoptosis was examined by agarose gel electrophoresis and TUNEL's method. The mRNA levels of Bax and Bcl-2 were determined by RT-PCR and the protein expression of phosphorylated and total Akt were assessed by Western blot.

Results Losartan-treated CHF rats had lower LVEDP $(p<0.01)$, higher LVEF $(p<0.05)$, lower plasma NE $(p<0.05)$ and myocardium Ang II $(p<0.05)$, but higher plasma Ang II $(p<0.05)$ than vehicletreated CHF rats. Losartan-treated CHF rats had no obviously "DNA ladder" which was the character of apoptosis, and the apoptosis index was also reduced $(p<0.05)$ with a lower expression of Bax/ $\mathrm{Bcl}-2$ gene $(\mathrm{p}<0.05)$ and a higher protein expression of $\mathrm{p}-\mathrm{Akt}(\mathrm{p}<0.05)$. Conclusion Losartan might inhibit cardiomyocyte apoptosis and improve cardiac function in aortic banded rats by blocking Ang II to bind AT1-R and promoting the activation of Akt.

\section{e0023 THE STUDY FOR RELATIONSHIP BETWEEN THE ANGIOTENSIN II TYPE 1 RECEPTOR GENE POLYMORPHISM AND ESSENTIAL HYPERTENSION IN KAZAKANS OF XINJIANG}

doi:10.1136/hrt.2010.208967.23

${ }^{1} \mathrm{Li}$ Nanfang, ${ }^{2} \mathrm{Li}$ Tao, ${ }^{3}$ Wang Xinling, ${ }^{4} \mathrm{Zu}$ Feiya, ${ }^{2}$ Wang Jun, ${ }^{3} \mathrm{Z}$ hang Delian, ${ }^{4}$ Zhou Keming. ${ }^{1}$ Hypertension Unit of The People's Hospital of Xinjiang Uygur Autonomous Region, The Institute of Hypertension of Xinjiang, The Center of Hypertension of The People's Hospital of Xinjiang Uygur Autonomous Region; ${ }^{2}$ Hypertension Unit of the People's Hospital of Xinjiang Uygur Autonomous Region; ${ }^{3} T$ The Institute of Hypertension of Xinjiang; ${ }^{4}$ The Center of Hypertension of The People's Hospital of Xinjiang Uygur Autonomous Region

Objective To investigate the relationship between the A1166C allele polymorphism of angiotensin II Type 1 Receptor (AT1R) gene and the essential hypertension in Kazakans of Xinjiang.

Methods PCR and restriction fragment length polymorphism methods (PCR-RFLP) were used to detect the A1166C polymorphism of AT1R gene in Kazakans including 321 patients with hypertension and 203 normotensive controls. The frequencies of genotype distribution hypertensives and normotensives were studied.

Results The genotype frequencies of A1166C were AA 0.7664, AC 0.2274 , CC 0.0062 in the hypertension group and the corresponding frequencies were $0.7537,0.2463,0$ in the control group respectively; The A1166 and 1166C allele frequencies were 0.8801, 0.1199 respectively in hypertension group and $0.8768,0.1232$ in normotensive group; The distribution of A1166C-genotype and the allele frequencies of A1166/1166C were not statistically significant in hypertension group as compared to normotensive group ( $p>0.05)$.

Conclusion There was no significant association found between the A1166C polymorphism of AT1R gene and essential hypertension in Kazakans of Xinjiang. Abbreviations: Angiotensin II Type 1 Receptor (AT1R).

\section{Q0024 ALDEHYDE DEHYDROGENASE-2 G1951A GENE POLYMORPHISM AND DRINKING BEHAVIOUR IN MALES}

doi:10.1136/hrt.2010.208967.24

${ }^{1}$ Yang Jing, ${ }^{2} \mathrm{Li}$ Nanfang, ${ }^{3} \mathrm{Li}$ Tao, ${ }^{4} \mathrm{Cao}$ Mei, ${ }^{2}$ Shao Liang, ${ }^{3} \mathrm{Ma}$ Yonghua, ${ }^{4} \mathrm{Hu}$ Junli. ${ }^{1}$ Hypertension Unit of The People's Hospital of Xinjiang Uygur Autonomous Region, The Institute of Hypertension of Xinjiang, The Center of Hypertension of The People's Hospital of Xinjiang Uygur Autonomous Region; ${ }^{2}$ Hypertension Unit of The People's Hospital of Xinjiang Uygur Autonomous Region; ${ }^{3}$ The Institute of Hypertension of Xinjiang; ${ }^{4}$ The Center of Hypertension of The People's Hospital of Xinjiang Uygur Autonomous Region

Objective To study the distribution of genotypes about aldehyde dehydrogenase- $2\left(\mathrm{ALDH}_{2}\right)$ and its relationship with drinking behaviours in males.

Methods 226 males were enrolled for collecting blood samples and data about drinking behaviours. $\mathrm{ALDH}_{2}$ genotypes were detected by PCR-restriction fragment length polymorphism (PCR-RFLP).

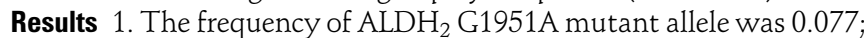
2. The frequency of $\mathrm{ALDH}_{2}$ mutant genotypes (GA+AA) was significantly lower in males whose daily alcohol consumption $\geq 32.83 \mathrm{~g}$ (mean of daily alcohol consumption) than those of $<32.83 \mathrm{~g}(\mathrm{p}<0.05)$. Comparing with GG, the subjects with $\mathrm{ALDH}_{2}$ mutant genotypes $(\mathrm{GA}+\mathrm{AA})$ were significantly lower in alcohol consumption per occasion (Chinese white liquor), daily alcohol consumption and cumulative alcohol consumption, and had a shorter drinking years $(p<0.05)$. Conclusion $\mathrm{ALDH}_{2}$ G1951A Polymorphism was found in males of Xinjiang, and it was associated with drinking behaviour.

\section{e0025 ALDEHYDE DEHYDROGENASE-2 G1951A GENE POLYMORPHISM AND OBSTRUCTIVE SLEEP APNOEA SYNDROME IN MALE DRINKERS}

doi:10.1136/hrt.2010.208967.25

${ }^{1}$ Yang Jing, ${ }^{2}$ Li Nanfang, ${ }^{3}$ Zhou Ling, ${ }^{4}$ Shao Liang, ${ }^{2}$ Cao Nei, ${ }^{3}$ Wang Yingchun, ${ }^{4}$ Xiang Hong. ${ }^{1}$ Hypertension Unit of The People's Hospital of Xinjiang Uygur Autonomous Region, The Institute of Hypertension of Xinjiang, The Center of Hypertension of The People's Hospital of Xinjiang Uygur Autonomous Region; ${ }^{2}$ Hypertension Unit of The People's Hospital of Xinjiang Uygur Autonomous Region; ${ }^{3}$ The Institute of Hypertension of Xinjiang; ${ }^{4}$ The Center of Hypertension of The People's Hospital of Xinjiang Uygur Autonomous Region

Introduction To study the relationship between aldehyde dehydrogenase-2 $\left(\mathrm{ALDH}_{2}\right)$ G1951A gene polymorphism and Obstructive Sleep Apnoea Hypopnoea Syndrome (OSAHS) in male drinkers. 